\title{
Syndromic Surveillance Practice in the United States 2014: Results from a Nationwide Survey
}

\author{
Tera Reynolds ${ }^{\star 1}$, Scott Gordon², Paula Soper ${ }^{2}$, James Buehler ${ }^{3}$, Richard Hopkins ${ }^{4}$ and \\ Laura Streichert ${ }^{1}$
}

${ }^{1}$ ISDS, Boston, MA, USA; ${ }^{2}$ ASTHO, Arlington, VA, USA; ${ }^{3}$ Philadelphia Department of Public Health, Philadelphia, PA, USA; ${ }^{4}$ University

of Florida, Tallahassee, FL, USA

\section{Objective}

To present the results of a nationwide survey designed to assess the syndromic surveillance (SyS) practices and capacity-building assistance (CBA) needs of U.S. state public health authorities (PHAs).

\section{Introduction}

Spurred by recent advances in PH informatics, the implementation of the Medicare and Medicaid Electronic Health Records Incentive Programs (Meaningful Use), and the opportunities provided by the availability of the redesigned BioSense program, SyS has become an increasingly important component of the biosurveillance enterprise. Knowing how and when jurisdictions use SyS, as well as challenges faced, allows ISDS, ASTHO, CDC, and other partners to provide relevant CBA - information transfer, training, and technical assistance - to further biosurveillance practice.

\section{Methods}

The survey instrument was developed with input from ISDS staff and Board members, ASTHO, and other stakeholders. After piloting, and revising based on feedback, the survey instrument was sent via email to a primary contact responsible for SyS or, for PHAs without a system, a knowledgeable contact, at 50 state, the District of Columbia (DC), and 6 territorial PHAs. Quantitative analyses were performed using R@. Results for territories, comparisons with earlier surveys, and mixed-method analyses will be included in the presentation.

\section{Results}

We received responses from practitioners in all 50 states and DC. Eighty percent of the 51 survey respondents report that they routinely access and review data for SyS and, of the 10 not currently practicing SyS, 6 plan to in the future.

Key findings (*NOTE: The Ns indicated below vary due to skip patterns in the survey and missing data):

- Of the 39 respondents conducting SyS, 13 are using BioSense only, 9 another application only, and 17 both.

- Among those that conduct SyS and do not use BioSense only:

- $100 \%$ monitor emergency department (ED) visits, and 52\% monitor poison control center calls $(n=27)$.

- The median percentage of ED facilities within a jurisdiction participating in SyS is $70 \%$ (range: $2-100 \%, \mathrm{n}=25$ ).

- 96\% report that the field on which their syndrome definition relies is chief complaints-free text, 50\% chief complaints-drop-down menu, and $46 \%$ diagnosis codes (select all that apply question; $\mathrm{n}=28$ ).

- Of the respondents using BioSense:

- 40\% report that BioSense is their primary SyS data acquisition, management, and analysis application $(\mathrm{n}=30)$. Of those using a local application and BioSense ( $\mathrm{n}=17), 6 \%$ report that BioSense is the primary application.

- $97 \%$ are currently, or planning to within the next year, sharing aggregate data with $\mathrm{CDC}$ and/or at least one other jurisdiction through BioSense $(n=30)$.
- $27 \%$ are currently sharing data at a visit record level with CDC and/or at least one other jurisdiction through BioSense $(n=30)$.

- Of those not currently sharing data at a visit record level through BioSense ( $n=22), 23 \%$ plan to in the future, $41 \%$ are unsure, and $36 \%$ do not intend to.

- $69 \%$ report that data from hospitals flow to their SyS system via direct connection from individual hospitals; $14 \%$ report that hospitals connect to BioSense, and the health department accesses via BioSense; and $11 \%$ indicated both of these options (Select all that apply question; $\mathrm{n}=36$ ).

- Of the respondents that conduct SyS and have data quality monitoring processes in place $(n=27), 85 \%$ report that their jurisdiction monitors data acquisition interface(s) at the transmission level on a routine basis.

- Of those conducting or using SyS:

- 58\% find the methods highly useful for trend analysis $(n=36)$, $51 \%$ for situational awareness during a PH event/emergency $(n=37)$, and $61 \%$ for influenza surveillance $(n=38)$.

- The median cost of materials and effort per 100,000 people is about $\$ 4,800$ (range: $\$ 18-\$ 18,900, \mathrm{n}=18$ ). *NOTE: Respondents were asked, to the extent possible, to exclude one-time development costs.

- The median number of FTEs dedicated to SyS (a) analysis and response and (b) technical system maintenance is 0.5 for each category (rangea: $0-7.83, \mathrm{n}=36$; rangeb: $0-6.83, \mathrm{n}=36$ ).

\section{Conclusions}

SyS practice continues to vary greatly between states in terms of adoption and resources allocated. The results of this survey provide a snapshot of the current SyS landscape, and are being used to inform the development of CBA activities at the state level.

For a more complete picture of the landscape, future work will include a survey of SyS practice among local PHAs.

\section{Keywords}

Challenges; Syndromic surveillance practices; Survey results

\section{Acknowledgments}

This work supported by CDC through a Cooperative Agreement to ASTHO.

\section{*Tera Reynolds \\ E-mail: treynolds@syndromic.org}

